\title{
Clasificación de los Puertos Españoles mediante Indicadores de Explotación utilizando Análisis de Conglomerados
}

\section{Classification of Spanish Ports by studying Operational Indicators using Cluster Analysis}

DOI: http://dx.doi.org/10.17981/ingecuc.12.2.2016.04

Artículo de Investigación Científica - Fecha de Recepción: 10 de Noviembre de 2015, Fecha de Aceptación: 7 de Abril de 2016

\begin{abstract}
Alfonso Camarero Orive*
Universidad Politécnica de Madrid. Escuela de Ingenieros de Caminos, Canales y Puertos. Departamento de Ingeniería Civil. Transporte y territorio. Madrid, España alfonso.camarero@caminos.upm.es
\end{abstract}

\author{
María del Mar Cerbán Jiménez \\ Universidad de Cádiz. Escuela Politécnica Superior. Departamento de Economía General \\ Algeciras, España mariadelmar.cerban@uca.es
}

Ignacio J. Turias Domínguez

Universidad de Cádiz. Escuela Politécnica Superior. Departamento de Ingeniería Informática

Algeciras, España ignacio.turias@uca.es

\section{Nicoletta González Cancelas}

Universidad Politécnica de Madrid. Escuela de Ingenieros de Caminos, Canales y Puertos. Departamento de Ingeniería Civil. Transporte y territorio. Madrid, España nicoleta.gcancelas@upm.es

\section{Alberto Camarero Orive \\ Universidad Politécnica de Madrid. Escuela de Ingenieros de Caminos, Canales y Puertos. Departamento de Ingeniería Civil. Transporte y territorio. Madrid, España alberto.camarero@upm.es}

Cómo citar este artículo:

A. Camarero Orive, M. Cerbán Jiménez, I. Turias Domínguez, N. González Cancelas y A. Camarero Orive, “Clasificación de los puertos españoles mediante indicadores de explotación utilizando análisis de conglomerados”, INGE CUC, vol. 12, No.2, pp.41-49, 2016. DOI: http://dx.doi.org/10.17981/ingecuc.12.2.2016.04

\begin{abstract}
Resumen- En este artículo se clasifican los puertos españoles utilizado una serie de indicadores que caracterizan la actividad portuaria. Estos indicadores se tratan con herramientas de análisis estadístico. Se ha elegido el análisis de conglomerados para realizar agrupamientos entre los puertos seleccionados. Para ello se ha seguido una metodología de trabajo que cubre todas las fases de la investigación: entorno, caracterización, fuente de datos, análisis de conglomerados, resultados y análisis de resultados. Los resultados obtenidos demuestran que los puertos españoles se pueden caracterizar correctamente por medio de indicadores físicos y de explotación, y que el análisis de conglomerados es una herramienta válida y útil para el entorno portuario.
\end{abstract}

Palabras clave- Puerto, clasificación, indicadores, análisis de conglomerados, explotación portuaria.

\begin{abstract}
This article classifies Spanish ports using a number of indicators that characterize the port activity. These indicators are treated with statistical analysis tools. Cluster analysis has been chosen to perform groupings among the selected ports. A methodology that covers all phases of research: environment, characterization, data source, cluster analysis, results and analysis of results have been followed in this paper. The results show that the Spanish ports are correctly characterized by physical and exploitation indicators, and that cluster analysis is a valid and useful tool for the port environment.
\end{abstract}

Keywords - Ports, classification, indicators, cluster analysis, port operations. 


\section{CLASIFICACIÓN DE LOS PUERTOS ESPAÑOLES MEDIANTE INDICADORES DE EXPLOTACIÓN UTILIZANDO ANÁLISIS DE CONGLOMERADOS}

\section{INTRODUCCIÓN}

En el entorno portuario se vienen realizando numerosos estudios basados en datos del tráfico marítimo y de las operaciones portuarias. Es común en estas investigaciones centrarse en criterios como la capacidad de la línea de atraque, la distribución de los muelles, la eficiencia en la operativa de carga/descarga, la seguridad, etc. Estos trabajos se suelen realizar con el propósito de conocer las ventajas e inconvenientes de un escenario concreto. El objetivo final de todos ellos es mejorar la operatividad y el rendimiento portuario, ya que estos informes sirven para diseñar estrategias de desarrollo.

El negocio marítimo portuario se presta al modelado analítico en todo el ciclo de gestión con casi todos los actores involucrados: administración, sociedades de clasificación, navieros, astilleros, terminales portuarias, aseguradoras y las mismas autoridades portuarias. Sin embargo, aún es notoria la falta de metodologías cuantitativas en la investigación del negocio; en buena parte, debido a la misma complejidad del sistema, a la gran cantidad de factores que intervienen y a los múltiples criterios que se pueden aplicar [1].

En este artículo se ha partido de otro punto de vista, al tratar los puertos no de manera individual, sino de manera conjunta. Es decir, se pretende compararlos entre sí, con base en algunos criterios definidos de antemano, mediante el uso de indicadores que puedan caracterizar un puerto.

El objetivo de este artículo es clasificar los puertos utilizando análisis de conglomerados (clúster) con indicadores de explotación portuaria. Esta clasificación de agrupamientos se hace con base en la semejanza de los puertos considerados. Las agrupaciones resultantes deben reflejar la realidad del entorno portuario, teniendo presente que la clasificación es uno de los objetivos fundamentales de la ciencia, y en este sentido el análisis de conglomerados nos proporciona los medios técnicos para realizarla.

No se pretende establecer un ordenamiento de mayor a menor, ni tampoco establecer criterios de competitividad entre los puertos.

El análisis de conglomerados puede ser una manera sencilla, pero rigurosa, para realizar estudios de clasificación o de aproximación general para un estudio posterior más complejo de la realidad portuaria. Hay que resaltar que el análisis clúster no hace ninguna ponderación de las características observadas, ya que a todas se les otorga el mismo peso, solamente compara la semejanza en la composición relativa de estas características.

Se ha desarrollado una metodología de trabajo que comprende: análisis del entorno, caracterización de los puertos, búsqueda de fuentes de datos, estudio de las variables o indicadores, toma de datos, aplicación del análisis estadístico y revisión de los resultados obtenidos. En la etapa de caracterización de los puertos, para el análisis de los resultados y para poder interpretar los agrupamientos, se utilizó criterio experto de profesionales del entorno portuario que conocen tanto la actividad portuaria como su operativa.

Uno de los retos más interesantes que se plantea es conocer cómo se mide la actividad portuaria. Para ello los indicadores portuarios incluyen factores físicos, de explotación y económicos, entre otros. En definitiva, hay que definir un puerto según una serie de variables y que estas sean las mínimas posibles.

El proceso metodológico de elaboración de indicadores es una tarea compleja que no reviste un carácter universalmente válido, debido a que su composición surge de la existencia de determinadas variables representativas inherentes al universo de análisis considerado. Es importante recordar que los indicadores son aquellos atributos, relaciones o contextos que se seleccionan como relevantes para describir a las unidades de análisis, lo cuaal significa que siempre existe un criterio previo de clasificación que se emplea para elegir los indicadores.

Para llegar a resultados fiables utilizando análisis estadísticos es preciso determinar cuáles son los indicadores adecuados, porque si no es posible que los resultados sean desacertados. Estos deben representar los aspectos de la realidad que se desea medir. Es decir, si se pretende analizar el entorno de los contenedores, se debe elegir solo las variables que tengan que ver, directa o indirectamente, con el tema de los contenedores. Por otra parte, los indicadores que se emplean para el posterior análisis estadístico deben cumplir con las cualidades de ser objetivos, manejables, comparables y fiables, y se prefiere los que son imprecisos, redundantes o poco homogeneizados.

Posteriormente, la metodología planteada se ha llevado a la práctica en el sistema portuario español, a través de las 28 autoridades portuarias repartidas por todo el litoral. Se ha seleccionado indicadores que hacen referencia al dimensionamiento de los puertos (variables físicas) y a la explotación de los puertos (variables de explotación).

El resultado del análisis de conglomerados será el agrupamiento de las Autoridades Portuarias con base en la similitud de sus características.

En España, el organismo público Puertos del Estado pide a todas las autoridades portuarias que publiquen los resultados de sus actividades en unas memorias anuales [2]. Estas memorias siguen un esquema común e informan de los mismos datos, de manera que estos son accesibles para el público y pueden usarse para realizar estudios de investigación. 


\section{REVISIÓN LiteraRIA}

Las ciudades son cada vez más innovadoras y compiten entre sí para atraer habitantes, empresas, turistas o eventos. Por ello, ofrecen una mayor capacidad de control y gestión en temas clave como el transporte, el entorno natural y la seguridad [3]. Todos esos aspectos son medibles y comparables con los de otras ciudades del mundo; es decir, a través de los estudios de clasificación se estudia el posicionamiento y las estrategias de desarrollo.

Desde hace más de 30 años se realizan con bastante frecuencia estudios en las ciudades sobre indicadores, para realizar clasificaciones de todo tipo, con enfoques generales o específicos. Los más comunes están referidos a la calidad de vida, a la sostenibilidad, la publicidad, etc. La finalidad es utilizar esos resultados para definir puntos fuertes y débiles en las ciudades estudiadas, y establecer líneas de actuación para el futuro. Se trata de otra manera de definir la competitividad urbana [4].

El concepto de ciudades inteligentes (smart cities), que en un principio fueron llamadas ciudades digitales, se ha ampliado y está siendo utilizado exitosamente en la planificación de las ciudades para impulsar y defender mejor el posicionamiento de sus estrategias urbanas. La imagen que transmiten las ciudades [5] debe sintetizar positivamente la identidad de estas para atraer las inversiones productivas y el talento creativo en general. Además, las redes de conocimiento y las comunidades virtuales permiten una ilimitada capacidad de innovación en esta especie de laboratorios de progreso de la era digital.

Sin embargo, los estudios o trabajos de investigación con indicadores en los puertos son bastante recientes y mucho menos variados, aunque en los últimos años se ha visto un avance importante de los trabajos que analizan la eficiencia y la productividad del sector portuario.

Doerr y Sánchez [6] realizaron un estudio sobre indicadores de productividad para la industria portuaria en América Latina para evaluar el estado de situación y las tendencias que experimenta.

Cerbán [7] ya realizó un estudio sobre los factores determinantes de la competitividad y competencia portuaria más relevantes en los puertos españoles a través de un grupo de indicadores sobre infraestructuras y servicios portuarios.

Mientras que algunos trabajos se han ocupado de obtener los indicadores adecuados para medir la eficiencia y productividad en el entorno portuario [8], otros estudios más específicos [9] se han centrado en el modelo de eficiencia de las terminales de contenedores. Todos estos estudios siempre tienen en cuenta que los puertos cumplen un rol para el desarrollo del comercio y en general para la competitividad del país, especialmente en el contexto de globalización que se ha experimentado en las últimas décadas.
En España se han realizado estudios relacionados con la competitividad que resaltan los factores que influencian la selección de un puerto en el sistema español [10], es decir, que reflejan la capacidad de captación de tráficos del sistema portuario español. España es el país de la Unión Europea que cuenta con mayor longitud de costa $(8000 \mathrm{~km}$.), y disfruta de una situación geográfica próxima al eje de importantes rutas marítimas con África, América y Asia.

En cuanto al análisis de conglomerados, se puede resumir diciendo que trata de obtener clasificaciones de grupos homogéneos, de acuerdo con semejanzas en las características evaluadas.

Un clúster es un conjunto de objetos que contiene datos similares y distintos a los de otro clúster. Se trata de una técnica descriptiva usada en la minería de datos, muy eficaz para encontrar patrones.

Los trabajos de Sokal y Sneath [11] marcaron hace años el inicio de los agrupamientos, que se han extendido por todos los ámbitos científicos.

Debido a la proliferación de datos y al acceso a los mismos, ha sido posible que la mayoría de las investigaciones analicen grandes conjuntos de datos, utilizando para ello las técnicas multivariantes.

El análisis de conglomerados (clustering) se ha venido utilizando en muchos entornos de investigación los últimos años, y también se relaciona con las redes neuronales artificiales y con la minería de datos [12]. Se presenta en áreas tan dispares como el reconocimiento de patrones [13], el análisis de datos [14], análisis médicos [15], económicos [16] o estudios ambientales para el análisis de patrones de distribución de especies [17]. En la enseñanza también se emplea para encontrar patrones de comportamiento del alumno para conocer su estilo de aprendizaje [18]. En los estudios acerca de las ciudades [19] también es frecuente utilizar esta herramienta de análisis. Incluso se encuentra referido a la competitividad de las empresas [20].

Medal y Sala realizaron en 2007 una clasificación del sistema portuario español basada en el análisis clúster. En su propuesta tomaron tan solo tres variables de entrada referidas al tráfico de graneles, sólidos y líquidos, y mercancía general [21], y concluyen que el sistema portuario español no era un conjunto homogéneo, sino que presentaba estructura de grupos dependiendo del volumen de la actividad de los puertos.

Respecto a los indicadores portuarios, existen algunas referencias que hay que considerar. El análisis DEA (Data Envelopment Analysis), o Análisis Envolvente de Datos aplicado al tráfico portuario se basa en diversos estudios ([22]- [24]). El DEA es un procedimiento no paramétrico que utiliza una técnica de programación lineal. Es una técnica de medición de la eficiencia de unidades productivas basada en la obtención de una frontera de eficiencia a partir de un conjunto de observaciones, sin necesidad de asumir ninguna forma funcional entre input o insumos y ouputs o productos. 
La estimación econométrica de funciones y distancias tratada para puertos ([25]- [28]) es una estimación empírica de funciones de costes portuarias, que tiene su origen en la década de los años setenta con el trabajo de Wanhill [29], cuyo objetivo era diseñar un modelo que permitiera determinar el número de atraques óptimos que minimiza el coste total de uso del puerto, entendiendo como tal la suma de dos componentes: el coste de suministrar la infraestructura (el atraque) y el coste del tiempo del barco en el puerto. En ese trabajo se considera que la inversión y planificación futuras han de hacerse teniendo en cuenta que los servicios portuarios no se pueden almacenar y, por tanto, hay un trade-off, o concesión mutua, entre el coste de la capacidad portuaria y el coste de permanencia de los barcos en el puerto (tiempo de servicio más tiempo de espera), que es determinante y ha de ser tenido en cuenta en el proceso de planificación.

\section{Metodología}

En primer lugar, se deben buscar variables representativas relacionadas con la actividad portuaria. Este proceso de identificación de variables, selección y posterior asignación de valores es fundamental porque estos indicadores son los que tienen que definir la actividad de un puerto de forma objetiva. La finalidad de este proceso de búsqueda de variables es encontrar el menor número de variables necesarias para caracterizar la actividad comercial de un puerto. Es preciso señalar que en los grandes puertos se dan cita distintas actividades de tráfico que se realizan en muelles especializados. Es decir, en un puerto puede existir a la vez tráfico de cruceros, contenedores o pesca que debe quedar reflejado en su caracterización.

Después de obtener los valores para los indicadores se pasa al análisis estadístico por conglomerados. El resultado de este análisis mostrará los agrupamientos que se han generado con esos indicadores de acuerdo con la semejanza entre puertos.

El análisis de conglomerados jerárquico es una herramienta de exploración diseñada para revelar agrupaciones naturales (o conglomerados) dentro de un conjunto de datos que de otro modo no serían evidentes. Es más útil cuando se desea agrupar un número pequeño (menos de unos pocos cientos) de objetos.

Para formar los conglomerados, el procedimiento comienza con cada observación en grupos separados. Después combina las dos observaciones que fueron las más cercanas para formar un nuevo grupo. Después de recalcular la distancia entre grupos, se combinan los dos grupos ahora más cercanos. Este proceso se repite hasta que quedan solamente el número de grupos que se haya elegido. Los objetos de cada grupo que se obtengan deben tener la mínima diferencia entre sí y la máxima con los objetos de otros grupos.
El clustering es muy sensible a la presencia de objetos atípicos, que inevitablemente serán los últimos en agrupar. Este procedimiento identifica claramente las relaciones multivariadas que difícilmente son accesibles a partir del análisis de las unidades espaciales individuales.

Para el análisis clúster se parte de una matriz ( $n$ $\mathrm{x} k$ ) de $n$ objetos (puertos) y $k$ variables (indicadores portuarios), donde cada objeto queda definido por el valor de sus variables.

\begin{tabular}{|c|c|c|c|c|}
\hline & $\mathrm{I}_{1}$ & $\mathrm{I}_{2}$ & $\ldots$ & $\mathrm{I}_{\mathrm{k}}$ \\
\hline $\mathrm{P}_{1}$ & $\mathrm{X}_{11}$ & $\mathrm{X}_{12}$ & $\ldots$ & $\mathrm{X}_{1 \mathrm{k}}$ \\
\hline $\mathrm{P}_{2}$ & $\mathrm{X}_{21}$ & $\mathrm{X}_{22}$ & $\ldots$ & $\mathrm{X}_{2 \mathrm{k}}$ \\
\hline$\ldots$ & $\ldots$ & $\ldots$ & $\ldots$ & $\ldots$ \\
\hline $\mathrm{P}_{\mathrm{n}}$ & $\mathrm{X}_{\mathrm{n} 1}$ & $\mathrm{X}_{\mathrm{n} 2}$ & $\ldots$ & $\mathrm{X}_{\mathrm{nk}}$ \\
\hline
\end{tabular}

El dendrograma es la representación gráfica, en forma de árbol jerárquico, que indica secuencialmente las uniones de las unidades espaciales, el cual permite definir el punto de corte de mayor aptitud y, con ello, la regionalización, donde se alinean en un eje todos los objetos y en el otro eje la distancia que les separa, lo que implica un nivel de jerarquía.

El historial de conglomeración muestra los niveles de fusión (coeficientes) en cada etapa. Los coeficientes muestran las distancias encontradas a las que se van uniendo los objetos, y elige siempre la mínima. El clúster formado en cada etapa se va modificando en las siguientes etapas al añadirse nuevos objetos. Observando los niveles de fusión y el diagrama de dispersión de los objetos se elegirá el número de clúster.

Como aplicación de esta metodología se ha realizado el estudio en el sistema portuario de titularidad estatal en España, de manera que no existen condicionantes previos sobre los puertos escogidos ni su elección responde a criterios orientados de antemano hacia este estudio. Se han recogido los datos directos de las Memorias de las Autoridades Portuarias y de los Anuarios Estadísticos de Puertos del Estado [2].

Atendiendo al tipo de información facilitada por los indicadores, estos se pueden clasificar como cuantitativos o cualitativos. Es decir, a los numéricos se les puede asignar un valor, como por ejemplo, el número de contenedores movidos al año, mientras que los descriptivos son los que explican, por ejemplo, qué actuaciones se han llevado a cabo para controlar un problema.

Hay que señalar que en esta metodología no se usa la ponderación en la importancia de los indicadores. Es decir, se considera que todos los indicadores tienen la misma influencia. Debido a que puede existir alguna dependencia indirecta entre los indicadores, es importante la correcta elección de estos para medir la actividad portuaria.

El siguiente esquema (fig. 1) presenta de forma detallada las fases y etapas que se han seguido en la metodología utilizada para la clasificación de los puertos españoles. 


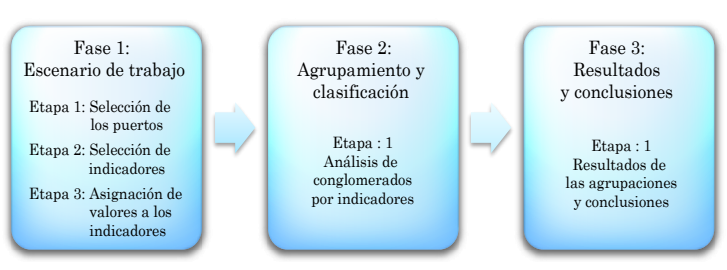

Fig. 1. Fases de la metodología de cálculo para clasificación. Fuente: Elaboración propia.

Fase 1. Escenario de trabajo. Define el escenario donde se realiza la investigación; para lo cual es preciso establecer a quién va dirigido el estudio y saber cuáles son las condiciones requeridas que se deben cumplir. Saber qué se va a medir y cómo.

Etapa 1. Selección de los puertos. Acota el escenario de trabajo y delimita las condiciones que deben cumplir los puertos del estudio, como por ejemplo, tamaño de las terminales o el tipo de tráfico de mercancías. El objetivo es contar con suficiente número de puertos para poder usar análisis estadísticos de datos.

Etapa 2. Selección de los indicadores. Estos indicadores deben caracterizar la actividad portuaria que se va a estudiar en base a la información disponible y a los requerimientos del análisis estadístico.

Etapa 3. Asignación de valores a los indicadores. Todos los indicadores deben tomar un valor concreto para cada puerto considerado en el estudio y garantizar la objetividad de dichos valores.

Fase 2. Agrupamiento y clasificación. El procedimiento multivariado de análisis de conglomerados es utilizado para obtener grupos homogéneos de unidades espaciales y maximizar la heterogeneidad entre los grupos formados, con los valores de los indicadores seleccionados.

Fase 3. Resultados y conclusiones. El resultado del análisis es obtener clasificaciones, con un marcado carácter exploratorio. De manera que los individuos pertenecientes a un grupo sean tan similares entre sí como sea posible, mientras que los otros grupos sean tan disimilares como sea posible.

\section{Caso práctico: aplicación a los puertos españoles}

Como aplicación de la metodología propuesta se ha hecho un estudio de agrupamiento sobre los puertos españoles. El sistema portuario español se compone de 28 autoridades portuarias que gestionan 46 puertos de interés general en un régimen avanzado de autonomía de gestión. Las terminales se explotan mediante concesiones, pudiendo realizarse también mediante concesión de obra pública. Además, las terminales del sistema portuario español son muy heterogéneas debido a la diferente tipología de sus tráficos, la diversidad de sus equipos de manipulación de mercancías y la variedad en su gestión y explotación [30], [31].

\section{Resultados}

Escenario de trabajo. Se eligieron las 28 autoridades portuarias españolas (fig. 2 y tabla 1 ).

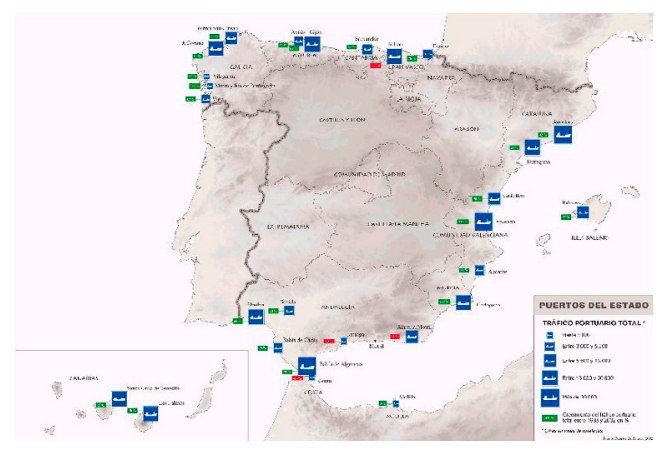

Fig. 2. Autoridades Portuarias en España. Fuente: Puertos del Estado.

Tabla 1. Autoridades Portuarias de España

\begin{tabular}{|l|l|l|}
\hline A Coruña & Castellón & Pasajes \\
\hline Alicante & Ceuta & $\begin{array}{l}\text { Santa Cruz } \\
\text { de Tenerife }\end{array}$ \\
\hline Almería & Ferrol-San Cibrao & Santander \\
\hline Avilés & Gijón & Sevilla \\
\hline Bahía de Algeciras & Huelva & Tarragona \\
\hline Bahía de Cádiz & Las Palmas & Valencia \\
\hline Baleares & Málaga & Vigo \\
\hline Barcelona & Marín y Ría de Pontevedra & Vilagarcía \\
\hline Bilbao & Melilla & \\
\hline Cartagena & Motril & \\
\hline
\end{tabular}

Fuente: Elaboración propia.

Indicadores. Los indicadores seleccionados para el análisis son los más comúnmente usados en los estudios del sistema portuario. Esta definición es importante porque la clasificación resultante dependerá de las variables elegidas. Los indicadores fueron elegidos siguiendo las investigaciones realizadas sobre el sistema portuario, y detalladas en el apartado de revisión literaria. De este conjunto, contando con la participación de expertos del sistema portuario español, se han seleccionado los más adecuados para este estudio. El grupo de indicadores corresponde a indicadores genéricos que tienen que ver con el tipo de explotación portuaria: graneles sólidos, graneles líquidos, mercancía general, pesca y tráfico de pasajeros; y con las características físicas de los puertos: superficie, longitud de los muelles o número de grúas.

Estos indicadores caracterizan y definen la actividad portuaria según sus dimensiones y la tipología del tráfico que se presta en ellos. Se seleccionó 19 indicadores que recogen las principales características generales de los puertos. En la tabla 2 se enumeran 


\section{CLASIFICACIÓN DE LOS PUERTOS ESPAÑOLES MEDIANTE INDICADORES DE EXPLOTACIÓN UTILIZANDO ANÁLISIS DE CONGLOMERADOS}

los indicadores junto con su definición y las unidades en las que se miden. Se utilizó indicadores numéricos y no se hizo aproximaciones ni simplificaciones.

Tabla 2. Selección de Indicadores Portuarios

\begin{tabular}{|c|c|c|}
\hline $\begin{array}{l}\text { Nombre } \\
\text { indicador }\end{array}$ & Definición & Unidad \\
\hline $\begin{array}{l}\text { Superficie de } \\
\text { flotación }\end{array}$ & $\begin{array}{l}\text { Zonas portuarias formadas } \\
\text { por las superficies de agua por } \\
\text { donde se desplazan los buques } \\
\text { en el recinto portuario. }\end{array}$ & $\mathrm{m}^{2}$ \\
\hline $\begin{array}{l}\text { Calado superior } \\
\text { a } 4 \mathrm{~m}\end{array}$ & $\begin{array}{l}\text { Longitud total de muelles } \\
\text { con un calado superior a los } 4 \\
\text { metros. }\end{array}$ & $\mathrm{m}$ \\
\hline $\begin{array}{l}\text { Longitud de } \\
\text { muelles de } \\
\text { contenedores }\end{array}$ & $\begin{array}{l}\text { Longitud total de los muelles } \\
\text { dedicados al tráfico de } \\
\text { contenedores. }\end{array}$ & $\mathrm{m}$ \\
\hline $\begin{array}{l}\text { Longitud } \\
\text { de muelles } \\
\text { de graneles } \\
\text { líquidos }\end{array}$ & $\begin{array}{l}\text { Longitud total de los muelles } \\
\text { dedicados al tráfico de graneles } \\
\text { líquidos. }\end{array}$ & $\mathrm{m}$ \\
\hline $\begin{array}{l}\text { Longitud de } \\
\text { muelles de } \\
\text { tráfico de } \\
\text { pasajeros }\end{array}$ & $\begin{array}{l}\text { Longitud total de los muelles } \\
\text { dedicados al tráfico de pasajeros. }\end{array}$ & $\mathrm{m}$ \\
\hline $\begin{array}{l}\text { Longitud de } \\
\text { muelles de pesca }\end{array}$ & $\begin{array}{l}\text { Longitud total de los muelles } \\
\text { dedicados a la pesca }\end{array}$ & $\mathrm{m}$ \\
\hline $\begin{array}{l}\text { Superficie } \\
\text { terrestre de } \\
\text { almacenamiento }\end{array}$ & $\begin{array}{l}\text { Superficie total en el puerto } \\
\text { dedicada al almacenamiento de } \\
\text { mercancías. }\end{array}$ & $\mathrm{m}^{2}$ \\
\hline $\begin{array}{l}\text { Longitud de } \\
\text { viales }\end{array}$ & $\begin{array}{l}\text { Longitud de todos los viales } \\
\text { interiores del puerto }\end{array}$ & $\mathrm{m}$ \\
\hline $\begin{array}{l}\text { Capacidad de } \\
\text { almacenamiento } \\
\text { frigorífico }\end{array}$ & $\begin{array}{l}\text { Volumen del espacio dedicado } \\
\text { en el puerto al almacenaje } \\
\text { refrigerado. }\end{array}$ & $\mathrm{m}^{3}$ \\
\hline $\begin{array}{l}\text { Producción de } \\
\text { hielo }\end{array}$ & $\begin{array}{l}\text { Cantidad de hielo fabricado } \\
\text { cada día en el puerto }\end{array}$ & t/día \\
\hline $\begin{array}{l}\text { Número total de } \\
\text { grúas }\end{array}$ & $\begin{array}{l}\text { Número de grúas de todos los } \\
\text { tipos utilizadas para la carga y } \\
\text { descarga de buques. }\end{array}$ & $\mathrm{n}$ \\
\hline $\begin{array}{l}\text { Tráfico de } \\
\text { graneles } \\
\text { líquidos }\end{array}$ & $\begin{array}{l}\text { Cantidad total de graneles } \\
\text { líquidos que han circulado por } \\
\text { el puerto en el año. }\end{array}$ & $\mathrm{t}$ \\
\hline $\begin{array}{l}\text { Tráfico de } \\
\text { graneles sólidos }\end{array}$ & $\begin{array}{l}\text { Cantidad total de graneles } \\
\text { sólidos que han circulado por el } \\
\text { puerto en el año. }\end{array}$ & $\mathrm{t}$ \\
\hline $\begin{array}{l}\text { Tráfico de } \\
\text { mercancía } \\
\text { general }\end{array}$ & $\begin{array}{l}\text { Cantidad total de mercancía } \\
\text { general que ha circulado por el } \\
\text { puerto en el año. }\end{array}$ & $\mathrm{t}$ \\
\hline Contenedores & $\begin{array}{l}\text { Número total de contenedores } \\
\text { que ha pasado por el puerto en } \\
\text { el año. }\end{array}$ & TEU \\
\hline $\begin{array}{l}\text { Número de } \\
\text { buques }\end{array}$ & $\begin{array}{l}\text { Número total de buques que ha } \\
\text { pasado por el puerto en el año. }\end{array}$ & $\mathrm{n}$ \\
\hline $\begin{array}{l}\text { Número de } \\
\text { pasajeros }\end{array}$ & $\begin{array}{l}\text { Número total de pasajeros que } \\
\text { ha pasado por el puerto en el } \\
\text { año. }\end{array}$ & $\mathrm{n}$ \\
\hline Pesca capturada & $\begin{array}{l}\text { Cantidad total de pesca } \\
\text { capturada en el año. }\end{array}$ & $\mathrm{t}$ \\
\hline $\begin{array}{l}\text { Avituallamiento } \\
\text { combustible }\end{array}$ & $\begin{array}{l}\text { Cantidad de combustible } \\
\text { suministrado a los buques en el } \\
\text { puerto. }\end{array}$ & $\mathrm{t}$ \\
\hline
\end{tabular}

Fuente: Elaboración propia.
Antes de proceder al análisis clúster se debe establecer el número de patrones, la definición de proximidad, el tipo de clustering y la representación de los datos. Para los datos es necesario realizar una tipificación previa, puesto que cada variable se mide en distinta escala. En cuanto a la similitud entre los objetos de un clúster, se puede optar por medidas de correlación, distancia o asociación. Para datos métricos en escala de intervalo, como es este caso, una de las más apropiadas es la distancia euclídea al cuadrado.

Por su parte, el algoritmo utilizado para la obtención de los clúster está basado en procedimientos jerárquicos, y se prefiere el vecino más lejano porque implica un encadenamiento completo.

La tabla 3 presenta el historial del análisis indicando el número de etapas, los conglomerados que se crean y los coeficientes de distancia euclidea. El resultado de aplicar el análisis de conglomerados a los indicadores es el que se presenta en el dendrograma de la fig. 3.

Tabla 3. Historial de Conglomeración

\begin{tabular}{|c|c|c|c|c|c|c|}
\hline \multirow{2}{*}{ Etapa } & \multicolumn{2}{|c|}{ Conglomerado que se combina } & \multirow{2}{*}{ Coeficientes } & \multicolumn{2}{|c|}{\begin{tabular}{|c|} 
Etapa en la que elconglormerado \\
aparececeor primerera vez
\end{tabular}} & \multirow{2}{*}{$\begin{array}{l}\text { Próxima } \\
\text { etapa }\end{array}$} \\
\hline & \begin{tabular}{|c} 
Conglomerado \\
1
\end{tabular} & $\frac{\text { Conglomerado }}{2}$ & & 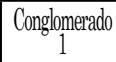 & \begin{tabular}{|c|c|} 
Conglomerado \\
2
\end{tabular} & \\
\hline 1 & 19 & 20 & 896 & 0 & 0 & 3 \\
\hline 2 & 2 & 17 & ,970 & 0 & 0 & 5 \\
\hline 3 & 19 & 28 & 1,501 & 1 & 0 & 7 \\
\hline 4 & 3 & 23 & 1,539 & 0 & 0 & 6 \\
\hline 5 & 2 & 24 & 2,627 & 2 & 0 & 7 \\
\hline 6 & 3 & 21 & 3,107 & 4 & 0 & 9 \\
\hline 7 & 2 & 19 & 3,246 & 5 & 3 & 9 \\
\hline 8 & 13 & 14 & 4,604 & 0 & 0 & 17 \\
\hline 9 & 2 & 3 & 5,105 & 7 & 6 & 10 \\
\hline 10 & 2 & 18 & 6,095 & 9 & 0 & 15 \\
\hline 11 & 9 & 25 & 6,951 & 0 & 0 & 13 \\
\hline 12 & 6 & 11 & 7,149 & 0 & 0 & 14 \\
\hline 13 & 9 & 10 & 10,071 & 11 & 0 & 16 \\
\hline 14 & 1 & 6 & 11,156 & 0 & 12 & 17 \\
\hline 15 & 2 & 12 & 11,693 & 10 & 0 & 19 \\
\hline 16 & 9 & 15 & 21,242 & 13 & 0 & 24 \\
\hline 17 & 1 & 13 & 24,660 & 14 & 8 & 19 \\
\hline 18 & 8 & 16 & 25,450 & 0 & 0 & 21 \\
\hline 19 & 1 & 2 & 29,170 & 17 & 15 & 20 \\
\hline 20 & 1 & 4 & 35,246 & 19 & 0 & 24 \\
\hline 21 & 8 & 26 & 36,114 & 18 & 0 & 23 \\
\hline 22 & 7 & 22 & 42,862 & 0 & 0 & 25 \\
\hline 23 & 5 & 8 & 54,482 & 0 & 21 & 27 \\
\hline 24 & 1 & 9 & 54,932 & 20 & 16 & 25 \\
\hline 25 & 1 & 7 & 72,255 & 24 & 22 & 26 \\
\hline 26 & 1 & 27 & 91,498 & 25 & 0 & 27 \\
\hline 27 & 1 & 5 & 159,242 & 26 & 23 & 0 \\
\hline
\end{tabular}

Fuente: Elaboración propia. 

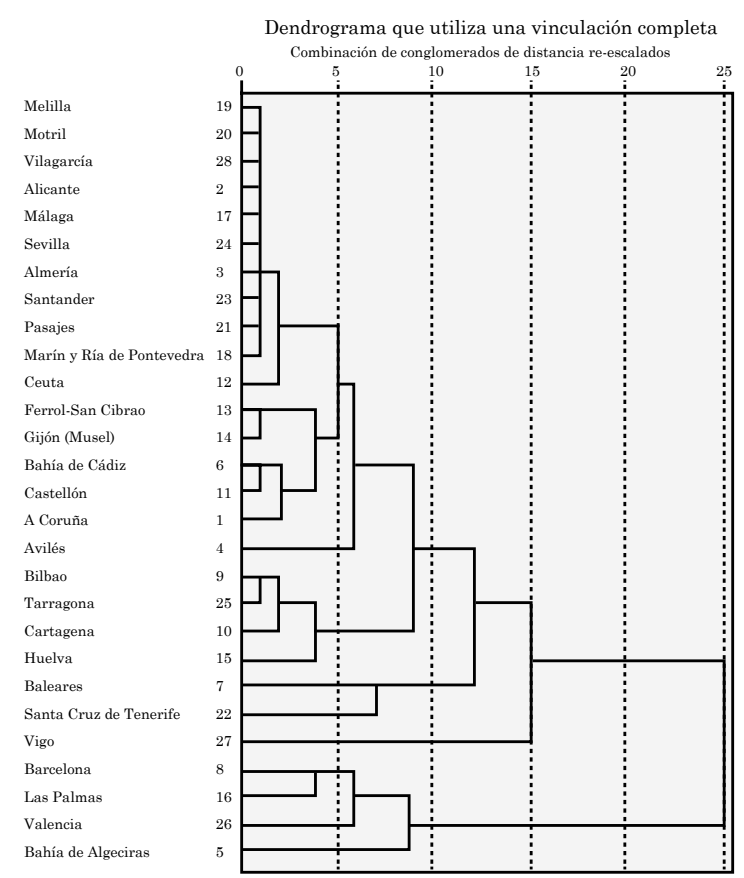

Fig. 3. Dendrograma con los indicadores portuarios. Fuente: Elaboración propia.

Análisis de los resultados en los puertos españoles. El examen de los resultados obtenidos con el agrupamiento de conglomerados es la última fase de la metodología y la que va a servir para validar la propuesta. A partir del dendrograma se pueden distinguir los agrupamientos.

En primer lugar se observan claramente diferenciados varios grupos (fig. 4). El primero está formado por Barcelona, Las Palmas, Valencia y Bahía de Algeciras, y el segundo grupo con el resto. Sin extendernos en muchas consideraciones, se puede añadir que ese primer grupo corresponde a los puertos con más tráfico de España. Son los puertos que tienen una mayor especialización en sus terminales y son los más relevantes en el tráfico internacional. Destacan en el tráfico de contenedores, en el avituallamiento de combustible y también en el tráfico de pasajeros. De este primer grupo, Algeciras es el puerto que más se separa de los demás, quizá por estar mucho más limitado en otras actividades portuarias que las mencionadas.

Del grupo restante se puede apreciar que el Puerto de Vigo también se diferencia del resto. Sus características hacen de él un puerto de importancia internacional en pesca y también formando parte de rutas internacionales de navegación.

El resto de los puertos presenta características más homogéneas y queda dentro del Grupo 3. Tienen similitudes en cuanto al tamaño, tráfico nacional e internacional, especialización, etc.

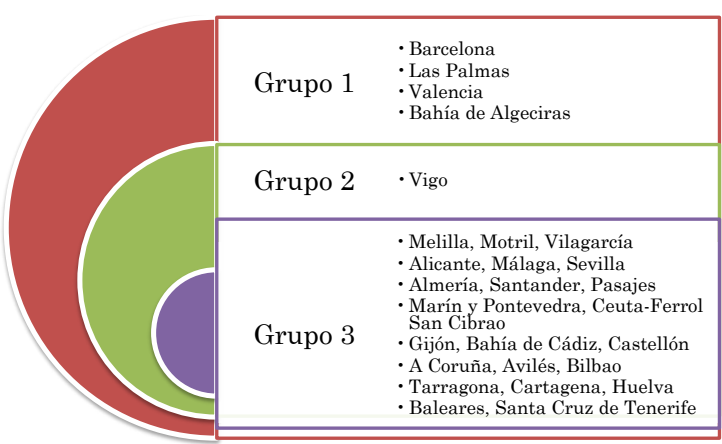

Fig. 4. Agrupamientos según el dendrograma. Fuente: Elaboración propia.

Sin embargo, este grupo 3 se puede seguir estudiando de la misma manera (fig. 5). En efecto, encontramos dos grupos diferenciados. El primero, Grupo 3_2, es el compuesto por Bilbao, Tarragona, Cartagena, Huelva, Baleares y Santa Cruz de Tenerife, que tienen un tamaño medio, forman parte del tráfico nacional e internacional y sus actividades son diversas, pero también especializadas.

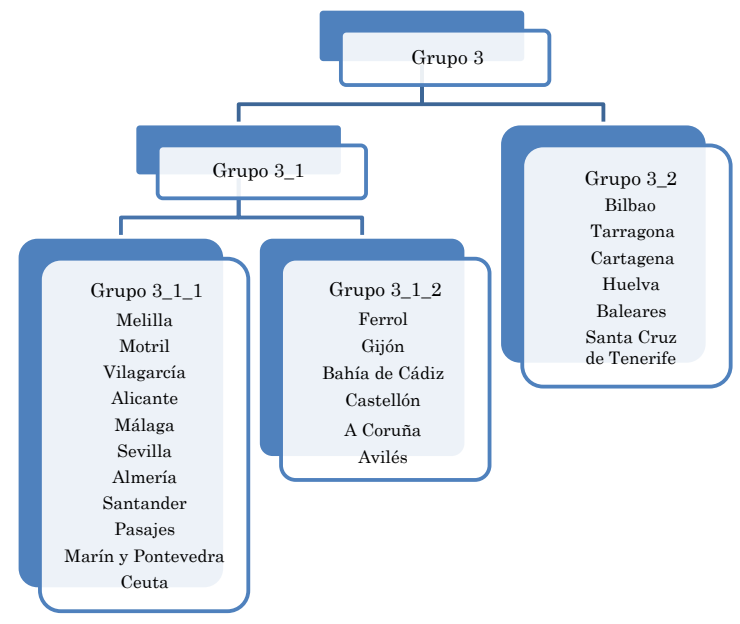

Fig. 5. Agrupamientos secundarios a partir del dendrograma Fuente: Elaboración propia.

El Grupo 3_1, a su vez, puede descomponerse en otros dos. De ellos, el Grupo 3_1_1 es el más numeroso con 11 unidades, pero también es el grupo más homogéneo de los encontrados y corresponde a los puertos menos especializados y de tamaño más modesto. En él se puede observar el mejor agrupamiento (lo que implica mayor similitud en sus características) de los puertos: Melilla, Motril, Vilagarcía, Alicante, Málaga, Sevilla, Almería, Santander, Pasajes, Marín-Pontevedra y Ceuta. Están más orientados a un tráfico nacional.

Este tipo de observaciones y conclusiones se pueden continuar y hacerse más detalladas bajando el nivel de corte en el dendrograma y observando los grupos que quedan. 


\section{CLASIFICACIÓN DE LOS PUERTOS ESPAÑOLES MEDIANTE INDICADORES DE EXPLOTACIÓN UTILIZANDO ANÁLISIS DE CONGLOMERADOS}

\section{Conclusiones}

En primer lugar hay que señalar que la metodología para realizar análisis de conglomerados con herramientas de clustering en el entorno portuario, utilizando indicadores que reflejen la actividad portuaria, es efectiva. Se puede afirmar que se puede definir indicadores a través de los cuales se refleje la actividad portuaria. Y con los valores que toman estos indicadores se puede realizar estudios de análisis estadísticos que permiten la agrupación de puertos con características similares de operación. El análisis de conglomerados es una herramienta que permite trabajar de manera sencilla, pero rigurosa, en el entorno portuario para realizar agrupamientos, comparaciones o clasificaciones.

Esta primera aproximación al análisis de los conglomerados no pretende encontrar variables de corte ni los valores de corte, sino comprobar que se agrupe de manera interpretable. Se debe elegir un número no muy pequeño de elementos para clasificar y definir los indicadores según el propósito de la investigación. Cuanto más específica sea la investigación, también lo serán los indicadores seleccionados. El número de indicadores tampoco debe ser muy alto para que la herramienta de análisis funcione en las mejores condiciones. Se estima que un número de entre 20 y 50 es apropiado.

En el caso práctico propuesto se demostró que los agrupamientos reflejan correctamente la realidad portuaria española. Sin duda, los grupos se pueden estudiar con más detenimiento para llegar a conclusiones más precisas, como pueden ser averiguar cuáles son los indicadores de corte y qué valores tienen.

En cuanto a las variables seleccionadas para este caso práctico, se considera que su número es adecuado, aunque también se podría añadir algunos nuevos indicadores que completen la información genérica de los puertos, por ejemplo, económicos o descriptivos que complementen la actividad portuaria. Asimismo, sería interesante conocer si el mismo patrón de clúster se puede obtener con un menor número de indicadores.

\section{FinANCIACIÓN}

Artículo de investigación derivado de la tesis doctoral titulada "Aplicación de indicadores de sostenibilidad medioambiental para la clasificación de puerto-verde en los puertos españoles" de la Universidad de Cádiz, España. Fecha de Inicio: Enero de 2013, Fecha de finalización: Enero de 2016.

\section{REFERENCIAS}

[1] M. Celik and Y I. Topcu, Analytical modelling of shipping business processes based on MCDM methods. Marit Policy Manage, vol. 36, no. 6, pp. 469-479, 2009. http://dx.doi. org/10.1080/03088830903346038

[2] Puertos del Estado. Memorias Anuales de Puertos del Estado, 2012.

[3] R. Giffinger, C. Fertner, H. Kramar, R. Kalasek, N. Pichler-Milanovic, and E. Meijers, Smart Cities - Ranking of European medium-sized cities. Smart cities-Ranking of European medium-sized cities 2007.

[4] A. Caragliu, C. Del Bo, and P. Nijkamp, Smart cities in Europe. : Vrije Universiteit, Faculty of Economics and Business Administration, 2009.

[5] N. Muñoz and M. Cervantes, Identidad, marketing de ciudades y marcas territoriales: place branding. Abaco: Revista de cultura y ciencias sociales, no. 60, pp. 27-47, 2009.

[6] O. Sanchez y R. Doerr, Indicadores de productividad para la industria portuaria: applicación en América Latina y el Caribe. : United Nations Publications, 2006.

[7] M. M. Cerbán, La competitividad portuaria de los puertos de titularidad estatal. Economistas, no. 116, pp. 277-282, 2008.

[8] J. C. Elizagárate Gutiérrez, P. Ruiz de Arbulo López, and P. Díaz de Basurto Uraga, "Competitividad del transporte multimodal a larga distancia: creación de un sistema de información para las decisiones estratégicas basado en indicadores," in XI Congreso de Ingeniería de Organización: Madrid, 5-7 de Septiembre de 2007, 2007, págs. 1747-1756, 2007.

[9] N. González, F Soler y A. Camarero, Modelo de eficiencia de las terminales de contenedores del sistema portuario español. Rect@: Revista Electrónica de Comunicaciones y Trabajos de ASEPUMA, no. 14, pp. 49-67, 2013.

[10] F. G. Laxe, M. JF. Seoane, C. P. Montes, Política y evaluación de la selección portuaria: el caso español. Revista de Evaluación de Programas y Políticas Públicas, vol. 1, no. 4, pp. 82-102, 2015. http://dx.doi.org/10.5944/ reppp.4.2015.13378

[11] R. R. Sokal and P. H. Sneath, Principles of numerical taxonomy. Principles of numerical taxonomy, 1963.

[12] J. Han, M. Kamber, and J. Pei, Data mining : concepts and techniques. Elsevier/Morgan Kaufmann, 2012. http:// dx.doi.org/10.1007/978-1-4419-1428-6 3752

[13] J. A. Moreiro González, "Aplicaciones al análisis automático del contenido provenientes de la teoría matemática de la información,” An. Doc., vol. 5, pp. 273-286, 2002.

[14] M. N. Arancegui, Análisis y políticas de clusters: teoría y realidad. Ekonomiaz: Revista vasca de economía, no. 53, pp.14-49, 2003.

[15] V. Moreno y X. Solé, Uso de chips de ADN (microarrays) en medicina: fundamentos técnicos y procedimientos básicos para el análisis estadístico de resultados. Med Clin, 122(Supl 1), pp. 73-79, 2004. http://dx.doi. org/10.1157/13057538

[16] Z. M. L. Martínez y I. R. S. Machado, Análisis del riesgo crediticio bancario en la economía cubana. Teoría y Praxis, no. 3, pp.77-87, 2007.

[17] J. 1. Field, K. Clarke, and R. Warwick, A practical strategy for analysing multispecies distribution patterns. Mar Ecol Prog Ser, no. 8, pp. 37-52, 1982.

[18] A.V. Arias, R. G. Cabanach, J. C. N. Pérez, J. M. S. Riveiro, I. P. Aguín, and S. R. Martínez, Enfoques de aprendizaje en estudiantes universitarios. Psicothema, vol. 12 , no. 3 , pp. 368-375, 2000.

[19] R. Méndez, J. J. Michelini, P. Romeiro, and S. Sánchez Moral, Ciudades intermedias y desarrollo territorial en Castilla-La Mancha, Xeográfica, no. 6, pp. 69-93, 2006.

[20] E. G. Erquiaga y J. C. A. Fernández, Factores de éxito y riesgo en la pyme: Diseño e implantación de un modelo para la mejora de la competitividad. Economía Industrial, no. 310 , pp. $149-161,1996$. 
[21] A. Medal y R. Sala, Clasificación y eficiencia del Sistema Portuario Español. XV Jornadas de ASEPUMA y III Encuentro Internacional Rect@ 2007.

[22] Y. Roll and Y. Hayuth, Port performance comparison applying data envelopment analysis (DEA). Maritime Policy and Management, vol. 20, no. 2, pp. 153-161, 1993. http://dx.doi.org/10.1080/03088839300000025

[23] J. Tongzon, Efficiency measurement of selected Australian and other international ports using data envelopment analysis. Transportation Research Part A: Policy and Practice, vol. 35, no. 2, pp. 107-122, 2001. http:/l dx.doi.org/10.1016/S0965-8564(99)00049-X

[24] M. Bonilla, T. Casasús, A. Medal, and R. Sala, "An efficiency analysis with tolerance of the Spanish port system," Int. J. Transp. Econ. / Riv. internazionale di Econ. dei Trasp., vol. 31, no. 3, pp. 379-400, 2004.

[25] C. Liu, H. Jula, and P. Ioannou, Design, simulation, and evaluation of automated container terminals. Intelligent Transportation Systems, IEEE Transactions on, vol. 3 , no. 1 , pp. $12-26,2002$.
[26] B. Tovar, S. Jara-Díaz y L. Trujillo, Funciones de producción y costes y su aplicación al sector portuario. Una revisión de la literatura. Documento de trabajo, no. 6, 2004.

[27] Marginal costs and scale economies in spanish ports, 25th European Transport Forum. Proceedings Seminar L, PTRC, London, 1997.

[28] B. Tovar, S. R. Jara-Diaz y L. Trujillo, Production and cost functions and their application to the port sector: a literature survey. World Bank Policy research working paper, no. 3123, 2003.

[29] S. R. C. Wanhill, A study in port planning: The example of Mina Zayed. Maritime Policy and Management, vol. 2, no. 1, pp. 48-55, 1974. http://dx.doi. org/10.1080/03088837400000033

[30] A. Camarero y N. González, Cadenas integradas de transporte. Fundación Agustín de Betancourt. Ministerio de Fomento, 2005.

[31] A. Camarero y N. González, Logística y transporte de contenedores. Fundación Agustín de Betancourt. Ministerio de Fomento, 2007. 